\title{
BreastCore
}

\section{Mastectomy - Current Challenges and Questions}

\author{
Bernd Gerber \\ Universitätsfrauenklinik und Poliklinik, am Klinikum Südstadt der Hansestadt Rostock, Rostock, Germany
}

As a result of mammography screening and neoadjuvant systemic treatment (NAST), more than $75 \%$ of all breast cancer cases in Germany are treated with breast-conserving surgery (BCS). Although contraindications for BCS like multifocality/multicentricity have been softened, mastectomy is still indicated in many patients. Mastectomy is not always performed as a simple mastectomy with axillary lymph node dissection. Today, nipple-/skin-sparing mastectomies (NSM, SSM) with autologous or heterologous reconstruction are used more frequently [1].

Simple mastectomy is a valid option in patients wishing to avoid repeat surgery due to affected margins or radiotherapy after BCS. Even in elderly or polymorbid patients with locally advanced breast cancer, mastectomy results in the rapid solution of certain problems (e.g. bleeding, smell, pain). On the other hand, the number of ipsilateral and prophylactic mastectomies (mostly SSM/NSM) is increasing steadily for no discernible reason [2]. Individual patient preferences should be integrated into treatment decisions. Thus, good communication is necessary to clarify with each patient the individual risk of recurrence and its impact in the setting of BCS compared to mastectomy.

A considerable number of affected patients - as well as many surgeons - believe that mastectomy is safer than BCS. However, it is well known that early breast cancer is a systemic disease. Depending on the intrinsic subtype, systemic treatment like chemotherapy, anti-HER2-directed treatment, and endocrine therapies are milestones in treatment that influence survival. Therefore, the impact of local treatment is less important for overall survival. In line with that, the margins for tumor resection declined from $2 \mathrm{~cm} 30$ years ago to no tumor cells on ink. The risk of local recurrence after BCS is further decreased by radiotherapy. While there is sufficient data showing that breast-conserving treatment is safe, new data suggest that the impact of axillary lymph node dissection on survival is less important than assumed in the past. Ongoing clinical trials are investigating whether even sentinel node biopsy could be avoided in early breast cancer [3]. With missing information regarding the involvement of axillary lymph nodes, radiotherapists must define new criteria for or against postmastectomy radiotherapy.
In the context of radiotherapy, we firstly have to consider psychological aspects of postmastectomy traumata, and secondly the type and timing of breast reconstruction.

Only few studies highlight the correlation of psychological wellbeing with surgical outcome. Outcomes concerning psychosocial aspects differ between breast cancer patients receiving mastectomy alone compared with those undergoing immediate or delayed reconstruction following mastectomy. If oncologically safe, there might be a psychological advantage in choosing immediate reconstruction. The most important reasons for that are the avoidance of postmastectomy traumata and the preservation of the naturallooking breast skin envelope.

Breast reconstruction after breast cancer and mastectomy is a sensitive topic, especially for younger women. Since breast cancer is a life-threatening disease, the timing of breast reconstruction should not interfere with the start of adjuvant treatment. In addition to patient wishes, their habitus, comorbidities, previous surgeries and planned radiation therapy need to be considered [4]. Reconstruction can be performed by employing heterologous or autologous procedures, both having advantages and disadvantages. With the refinements in surgical procedures, especially free flaps, and the development of supportive materials, the number of breast reconstructions is increasing in many countries. With respect to the higher cost of mastectomy without providing greater oncological safety compared to BCS, the question is whether health insurances should have to pay for a more cost-intensive treatment option?

Currently, the biggest controversy exists regarding prophylactic mastectomy in healthy, not breast cancer-affected women with or without $B R C A 1 / 2$ mutation and in unilaterally affected patients [5]. With the confirmation of a pathologic BRCA1/2 mutation, there is, for the first time, a chance of risk-reducing surgery in healthy women with an elevated risk of developing cancer. So far, a reduction in mortality by prophylactic surgery has only been proven for BRCA1/2 mutation carriers; this does not apply to other gene mutations. In healthy women with pathogenic $B R C A 1 / 2$ mutations, a risk-reducing bilateral mastectomy, mostly

\section{KARGER}

(๑) 2017 S. Karger GmbH, Freiburg

Fax +497614520714 
performed as NSM/SSM, leads to a verifiable reduction in mortality from breast cancer. This is particularly true for patients of a very young age. A further risk reduction is seen after bilateral oophorectomy. In affected BRCA1/2 mutation carriers, the recommendations for or against contralateral prophylactic mastectomy are controversial. This procedure reduces the incidence of later contralateral breast cancer; however, the effect on overall survival is not yet proven. Possibly only younger women with BRCA1/2 mutations and a prognostically favorable tumor biology (e.g. no chemotherapy indication, not triple-negative, not G3) may benefit from contralateral prophylactic mastectomy. A consensus statement from the American Society of Breast Surgeons states that BCS should even be offered to affected patients with $B R C A 1 / 2$ mutations after neoadjuvant chemotherapy and/or local oncoplastic procedures [6].

The pros and cons of prophylactic surgical options, different techniques, and alternatives ought to be explained to the patient and carefully discussed. In healthy BRCA1/2 mutation carriers, the alternative to prophylactic surgery is intensified early detection. No unequivocal data have been ascertained as yet concerning mortality from breast carcinoma.
This issue of BREAST CARE focusses on the most important issues concerning mastectomy: Max Dieterich and colleagues discuss different approaches to breast reconstruction [7], Anne-Sophie Heimes and colleagues highlight psychosocial aspects of immediate and delayed breast reconstruction [8], Christine Mau and Michael Untch ask for which patients prophylactic surgery is an option [9], and Fabian Riedel and colleagues discuss the oncological safety of BCS [10].

In conclusion, BCS is oncologically as safe as mastectomy and is associated with improved psychological well-being. BCS should be offered to all suitable patients, even after NAST. BCS has the best long-term cosmetic results with a low risk of complications. With the exception of locally advanced cancer or non-responders to NAST, there are only few indications for mastectomy, especially in the prophylactic setting in breast cancer-affected women. The detection of early breast cancer and more effective NAST will hopefully decrease the number of subsequent mastectomies in the future.

\section{Disclosure Statement}

The author declares no conflicts of interest.

\section{References}

1 Gerber B, Krause A, Dieterich M, et al.: The oncological safety of skin sparing mastectomy with conservation of the nipple-areola complex and autologous reconstruction: an extended follow-up study. Ann Surg 2009;249:461-468.

2 Wong SM, Freedman RA, Sagara Y, et al.: Growing use of contralateral prophylactic mastectomy despite no improvement in long-term survival for invasive breast cancer. Ann Surg 2017;265:581-589.

3 Reimer T, Stachs A, Nekljudova V, et al.: Restricted axillary staging in clinically and sonographically nodenegative early invasive breast cancer (c/i T1-2) in the context of breast conserving therapy: first results following commencement of the Intergroup-SentinelMamma (INSEMA) trial. Geburtsh Frauenheilk 2017; $77: 149-157$
4 Gerber B, Marx M, Untch M, et al.: Breast reconstruction following cancer treatment. Dtsch Arztebl Int 2015;112:593-600.

5 Ramaswami R, Morrow M, Jagsi R: Contralateral prophylactic mastectomy. N Engl J Med 2017;377:12881291.

6 Boughey JC, Attai DJ, Chen SL, et al.: Contralateral prophylactic mastectomy (CPM) consensus statement from the American Society of Breast Surgeons: data on CPM outcomes and risks. Ann Surg Oncol 2016;23: 3100-3105.

7 Dieterich M, Dragu A, Stachs A, Stubert J: Clinical approaches towards breast reconstruction: what is the appropriate reconstructive procedure for my patient? Breast Care 2017;12: DOI: 10.1159/000484926.
8 Heimes AS, Stewen K, Hasenburg A: Psychosocial aspects of immediate versus delayed breast reconstruction. Breast Care 2017;12: DOI: 10.1159/000485234.

9 Mau C, Untch M: Prophylactic surgery: for whom, when and how? Breast Care 2017;12: DOI: 10.1159/ 000485830.

10 Riedel F, Hennigs A, Hug S, Schaefgen B, Sohn C, Schuetz F, Golatta M, Heil J: Is mastectomy oncologically safer than breast-conserving treatment in early breast cancer? Breast Care 2017;12: DOI: $10.1159 / 000485737$. 